\title{
Analysis of 3-panel and 4-panel microscale ionization sources
}

\author{
Srividya Natarajan, ${ }^{1}$ Charles B. Parker, ${ }^{1, a)}$ Jeffrey R. Piascik, ${ }^{2}$ Kristin H. Gilchrist, ${ }^{2}$ \\ Brian R. Stoner, ${ }^{1,2}$ and Jeffrey T. Glass ${ }^{1}$ \\ ${ }^{1}$ Department of Electrical and Computer Engineering, Duke University, Durham, North Carolina 27708, \\ USA \\ ${ }^{2}$ Center for Materials and Electronic Technologies, RTI International, Research Triangle Park, \\ North Carolina 27709, USA
}

(Received 11 September 2009; accepted 21 April 2010; published online 21 June 2010)

\begin{abstract}
Two designs of a microscale electron ionization (EI) source are analyzed herein: a 3-panel design and a 4-panel design. Devices were fabricated using microelectromechanical systems technology. Field emission from carbon nanotube provided the electrons for the EI source. Ion currents were measured for helium, nitrogen, and xenon at pressures ranging from $10^{-4}$ to 0.1 Torr. A comparison of the performance of both designs is presented. The 4-panel microion source showed a $10 \times$ improvement in performance compared to the 3-panel device. An analysis of the various factors affecting the performance of the microion sources is also presented. SIMION, an electron and ion optics software, was coupled with experimental measurements to analyze the ion current results. The electron current contributing to ionization and the ion collection efficiency are believed to be the primary factors responsible for the higher efficiency of the 4-panel microion source. Other improvements in device design that could lead to higher ion source efficiency in the future are also discussed. These microscale ion sources are expected to find application as stand alone ion sources as well as in miniature mass spectrometers. (C) 2010 American Institute of Physics. [doi:10.1063/1.3429220]
\end{abstract}

\section{APPLICATIONS AND LITERATURE REVIEW OF MICROSCALE ION SOURCES}

\section{A. Introduction to ionization sources}

Ion sources are important scientific devices that find application in a variety of areas. They are used in particle accelerators, ion implantation systems for the semiconductor industry, semiconductor processes such as reactive ion etching, giant neutral beam injectors in experimental fusion reactor devices, and in analytical instruments such as mass spectrometers. ${ }^{1}$

Gas phase ionization techniques include electron ionization (EI), field ionization, and chemical (or charge transfer) ionization. Electron impact ionization or electron bombardment ionization is the process in which an electron with suitable energy collides with a neutral atom or molecule resulting in the formation of an ion and an electron. In field ionization, an electron is removed by tunneling from the molecule in the presence of a strong electric field, creating an ion. Field ionization tends to result in fewer fragmentation events than does EI. Chemical ionization uses a reagent gas, such as methane. The reagent gas is ionized using an EI source, for example, and then reacted with the analyte molecules, ionizing them in a lower energy state, also resulting in less fragmentation than in EI.

Liquid and solid phase ionization techniques are most commonly used for mass spectroscopy. Liquid phase techniques include atmospheric pressure chemical ionization, field desorption, and electrospray ionization (ESI). These techniques require the analyte compound to be nebulized

${ }^{a)}$ Electronic mail: cbparker@duke.edu. into the gas phase and the solvent removed through a series of stages of increasing vacuum (typically atmosphere to $10^{-6}$ Torr or greater) as the ions travel into the mass spectrometer. Solid phase techniques include matrix-assisted laser desorption/ionization (MALDI) and secondary ion mass spectrometry (SIMS). MALDI focuses short pulses of a laser on a surface that consists of a a solid or a viscous liquid matrix and the analyte, vaporizing the analyte and creating a plasma near the surface of the sample. In SIMS, a surface is irradiated by an ion beam and the resulting ions are analyzed. Finally, there are techniques such as direct analysis in real time (DART) and desorption ESI (DESI) that can work on liquid or solid surfaces. In DART, a stream of metastable ions are directed toward a surface, creating ions that are sampled. In DESI, a stream of ions is directed to a surface, desorbing ions from the surface. DART and DESI are atmospheric pressure techniques, so the generated ions are sampled through a series of differential pressured chambers into the mass analyzer. The other techniques mentioned are primarily vacuum techniques.

\section{Electron ionization}

Advantages of EI include a low energy spread for the resulting ions and easy design of the ion source. ${ }^{1}$ Disadvantages include low ion current and problems resulting from oxygen exposure and corrosion of the filament. EI is one of the most popular methods of ionization and is the method used in the miniature carbon nanotube (CNT)-based ionization sources in this study. In EI, the colliding electron is known as the primary electron, while the electron liberated from the gas molecule is known as the secondary electron. The primary electron needs a minimum amount of energy, 
called the ionization potential, to liberate the outermost electron from the gas atom. To achieve this energy, free electrons are accelerated through an appropriate voltage gradient. The EI cross-section is a measure of the probability that an electron collides with a neutral and produces an ion and is a function of electron energy and the properties of the neutral atom or molecule. ${ }^{1}$ The cross-section generally increases with electron energy at low energies, reaches a maximum around $70-200 \mathrm{eV}$ for most gases, and drops rapidly for higher electron energies. ${ }^{2}$

EI sources used in mass spectrometry require a welldefined electron beam and moderate ion currents. ${ }^{1}$ They typically operate at pressures ranging from $10^{-6}$ to $10^{-2}$ Torr, though some ion sources can operate at pressures as high as 0.75 Torr. $^{1}$ They can generate ion currents ranging from microampere to milliampere at these pressures.

Many EI sources use a cathode to emit electrons that bombard the neutral atoms or molecules and subsequently generate ions. The different types of cathodes typically used in EI sources are thermionic, or hot, cathodes; hollow cathodes; discharge cathodes; microwave cathodes; field emission cathodes, etc. ${ }^{1}$ Thermionic cathodes usually consist of a metal filament that emits electrons when heated to a sufficiently high temperature. ${ }^{1}$ In contrast to thermionic cathodes, cold cathodes are not heated thereby providing lower power consumption and minimal thermal load on the materials making up the cathode. For the most common type of cold cathode, field emission cathodes, a suitably high electric field is applied to the cathode to induce electron emission.

\section{B. Miniaturization of ion sources}

Reducing the size and cost of ion sources is an ongoing field of study that has demonstrated steady improvements. However, miniaturization in the present study means a radical reduction in size through application of a new technology and design trade-offs, particularly for the purpose of enabling a new instrument or application. Miniature sources typically have lower total ion current, a larger percent energy spread, and less collimation in the ion beam.

Many miniaturized ion sources use EI and have CNT field emitters as the cathode, ${ }^{3-8}$ or other cathode material. ${ }^{9}$ Field emission-based electron sources, also known as cold cathodes, consume less power and operate at lower temperatures than a comparable hot filament cathode. The lower power requirement makes cold cathodes particularly well suited to miniaturization. CNTs are well known for their field emission properties and are a natural choice for a miniaturized cold cathode. ${ }^{10,11}$ Recent publications have also documented the use of CNT-based EI sources as pressure gauges. ${ }^{12,13}$ King et al. ${ }^{14}$ are developing a miniature, high resolution reflectron time-of-flight mass spectrometer for in situ analysis of planetary atmospheres and are using a CNTbased field emission EI source because of its low power consumption. Velasquez-Garcia and Akinwande have reported using plasma enhanced chemical vapor deposition to grow CNTs for a microfabricated field ionization ion source for use in a portable microgas analyzer. ${ }^{15}$ Microplasmas or glow discharges are also common ion sources because these
A)

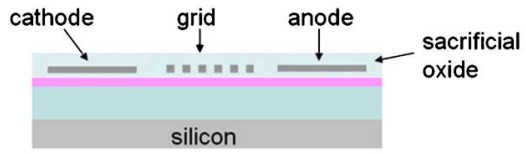

B)
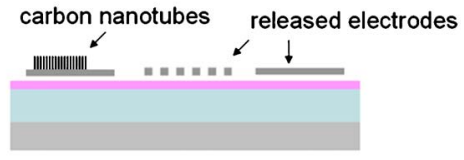

assembled triode

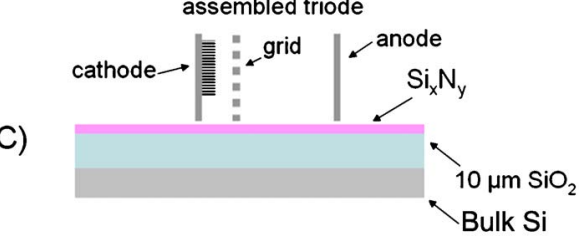

FIG. 1. (Color online) Process flow for MEMS device fabrication. The 10 $\mu \mathrm{m}$ thick oxide layer is below the silicon nitride layer and above the bulk silicon.

sources can be operated at atmospheric pressure. Hauschild and co-workers ${ }^{16,17}$ reported a fully integrated microfabricated mass spectrometer with an EI ion source. They reported ion currents in the 2 to $20 \mathrm{pA}$ range for pressures ranging from 7.5 to 750 mTorr. Rather than field emission, the source was a microplasma from which an electron beam was extracted.

\section{FABRICATION OF CNT-BASED MICROION SOURCES}

\section{A. Micro-Electro-Mechanical Systems (MEMS) processing}

The microion sources reported here were fabricated using MEMSCAP polysilicon multiuser MEMS process (PolyMUMPs). ${ }^{18}$ PolyMUMPS is a three-layer polysilicon surface micromachining process. The standard process includes a silicon nitride insulation layer that does not withstand high voltage operation. A significant enhancement in these devices was the inclusion of an insulating $\mathrm{SiO}_{2}$ layer to minimize leakage currents and thus maximize performance. ${ }^{4}$ A $10 \mu \mathrm{m}$ layer of high quality $\mathrm{SiO}_{2}$ was grown using thermal oxidation on the wafers prior to the standard MEMS process. The 3-panel microion source consists of microscale poly-Si panels as the cathode, grid, and ion collector. If the 3-panel source is operating in triode mode, rather than as an ion source, the voltage on the ion collector panel is changed from negative to positive and it used as an anode. Similarly, the 4-panel microion source consists of microscale poly-Si panels as the cathode, grid, anode, and ion collector. The process flow for the 3-panel device is shown in Fig. 1. The main steps involved in the process flow are explained in detail below.

After completion of the foundry process, the MEMS parts were soaked in HF to release the polysilicon electrode panels from the sacrificial oxide. Each MEMS part is provided with an integrated shadow mask of a specific pattern (blanket $/ 3 \times 3$ squares $/ 9 \times 9$ squares) during fabrication. An integrated shadow mask was used to selectively deposit the Fe catalyst in a patterned array on the cathode area using electron beam evaporation. Vertically aligned multiwalled 


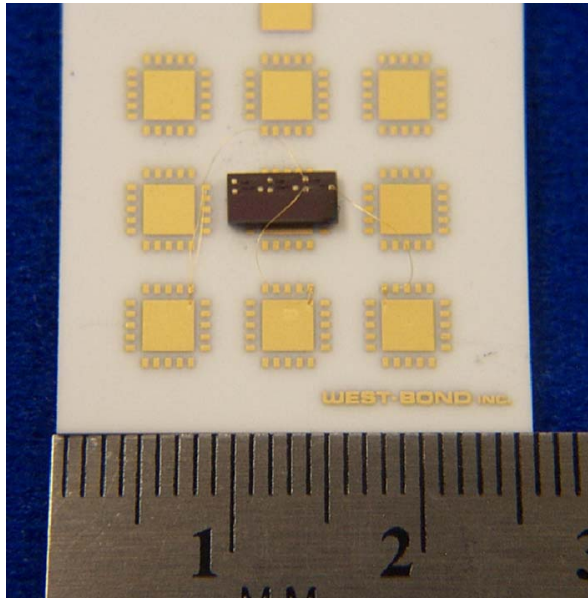

FIG. 2. (Color online) Three-panel device mounted and wire-bonded to a ceramic chip carrier.

CNTs (MWNTs) were deposited on the cathode using microwave plasma chemical vapor deposition ${ }^{19,20}$ with ammonia/ methane gas chemistry. Electron microscopy revealed MWNTs with an average diameter of approximately 30 to 50 $\mathrm{nm}$. The CNT length was controlled by varying the deposition time.

In order to provide electrical connections to the electrodes in each device, gold bond pads were deposited on the devices after the CNT growth. While the PolyMUMPs process provides gold metallization, the gold cannot withstand the CNT growth temperature. Therefore, electron-beam deposition was used to deposit gold bond pads through laserdrilled shadow masks after CNT growth. After bond pad deposition, the devices were assembled. Each of the electrodes, which were lying flat on the substrate, was manually rotated into a vertical, upright position. The electrodes have integrated polysilicon hinges and latches that support the panels in the vertical position. For the assembly, one micropipette was used to gently lift up the latch while a second micropipette was used to rotate the panel into a vertical position. The latch was then lowered onto the notches provided in the electrode panel to hold the latch in place.

After all panels were locked in place, the device was mounted and wire-bonded to a ceramic board for testing. A picture of a mounted and wire bonded device is shown in Fig. 2 in a ceramic chip carrier. Additional details of the device assembly process and integration of the ion source in a mass spectrometer are given elsewhere. ${ }^{21}$

\section{B. Device fabrication results}

A scanning electron microscope (SEM) micrograph of a fully assembled 3-panel device is shown in Fig. 3. The specific device characterized here had a cathode-to-grid spacing of $50 \mu \mathrm{m}$ before CNT deposition, a cathode-to-grid spacing of $30 \mu \mathrm{m}$ after CNT deposition, and a grid-to-collector spacing of $280 \mu \mathrm{m}$. The cathode is a $70 \times 70 \mu \mathrm{m}^{2}$ panel and the grid is a $3 \times 3$ array of $20 \times 20 \mu \mathrm{m}^{2}$ apertures, with a $2.5 \mu \mathrm{m}$ grid wire.

An SEM micrograph of an assembled 4-panel device is shown in Fig. 4. The CNT patterns were squares with

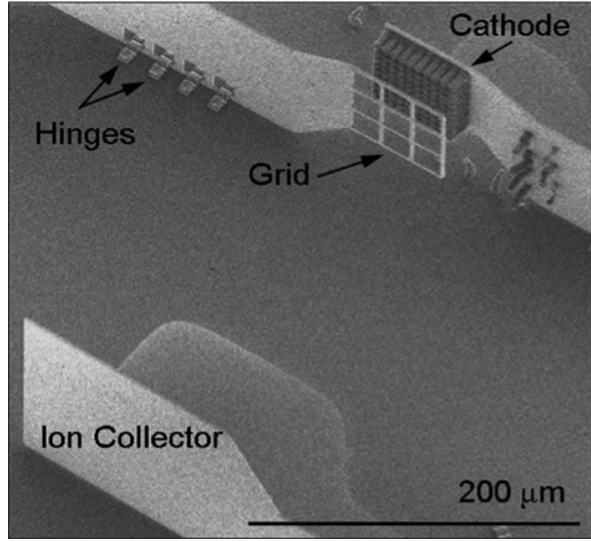

FIG. 3. SEM image of the 3-panel microion source which was used for SIMION simulations.

$10 \mu \mathrm{m}$ sides and a $3 \times 3$ grid of such patterns comprised the cathode. The cathode-to-grid spacing of this particular device was $50 \mu \mathrm{m}$ before CNT growth, and was reduced to $35 \mu \mathrm{m}$ after vertically aligned, $15 \mu \mathrm{m}$ CNTs were deposited on the cathode. The CNTs had an average diameter of 30-50 nm. The grid was a $3 \times 3$ array of $20 \times 20 \mu \mathrm{m}^{2}$ apertures with $2.5 \mu \mathrm{m}$ grid wire. The grid-to-anode spacing was $250 \mu \mathrm{m}$ and the anode had an area of $150 \times 150 \mu \mathrm{m}^{2}$. The ion collector panel had an area of $215 \times 150 \mu \mathrm{m}^{2}$ and stood $50 \mu \mathrm{m}$ away from the edges of the other 3-panels.

\section{PERFORMANCE COMPARISON AND ANALYSIS OF CNT-BASED MICROION SOURCES}

\section{A. Ionization in the microion sources}

\section{Ionization setup in the 3-panel microion source}

The 3-panel and 4-panel configurations are the two different microscale ion source designs studied here. The 3 -panel microion source is a microtriode structure operated under different voltage conditions. For the triode characteristics of this CNT-based device the reader is referred to a related publication. ${ }^{4}$ For typical triode operation, a positive voltage is applied to the grid and anode, while the cathode is kept at ground. This arrangement results in the field emission of electrons from the CNTs on the cathode. When operated in the saturation region (i.e., the region where the anode current has reached its maximum value), the microtriode typically generates electrons with energies in the 0 to $400 \mathrm{eV}$ range. When the triode operates in this region, most of the field

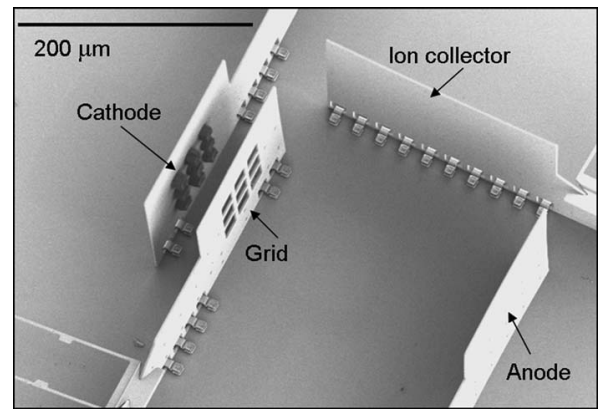

FIG. 4. SEM image of 4-panel microion source device that was used for SIMION simulations. 


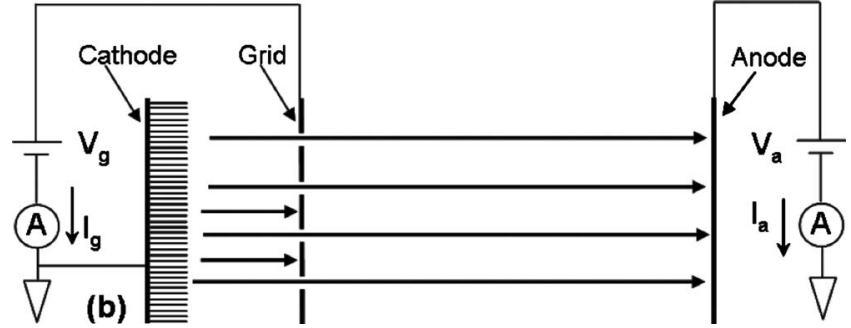

FIG. 5. Schematic diagram illustrating electron paths in the microtriode when operated as a conventional vacuum microelectronic three terminal device.

emission electron current is captured by the anode. A schematic illustrating the operation of the triode in this region is shown in Fig. 5.

The electrons generated by the cathode can be used to create ions via EI. This was achieved as follows in the 3-panel microion source/microtriode. The anode (now functioning as the ion collector) was made negative with respect to the grid electrode. Some fraction of the electron current passes through the openings in the grid, and continues toward the ion collector.

As the electrons approach the ion collector, the negative potential applied to the ion collector slows them down and forces them to reverse their direction back toward the grid. A schematic diagram illustrating the expected electron and ion paths in the 3-panel microion source is shown in Fig. 6. In order for the microtriode to generate ions, the grid and anode should be biased such that the electrons in the region between the grid and the anode are in the 0 to $200 \mathrm{eV}$ energy range. This energy range is suitable for ion generation via EI since EI cross-sections for most gases are highest in the 70 $200 \mathrm{eV}$ electron energy range. ${ }^{2}$ Biasing the electrodes appropriately ensures electron energies in this range so that ions are created between the grid and the ion collector.

\section{Ionization setup in the 4-panel microion source}

The design of the 4-panel microion source was based largely on the macroscopic ionization setup used by Smith and Tate. ${ }^{22}$ Ionization in the 4-panel device was achieved with the grid and anode electrodes at positive potentials compared to the cathode and ion collector. The ion collector was biased slightly negative with respect to the cathode so that the ion collector would be most likely to collect the (positive) ions that were generated. The electrons emitted from the CNT cathode via field emission were accelerated toward

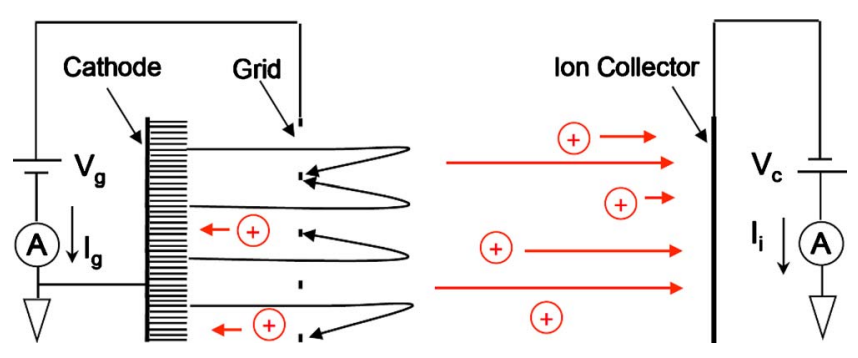

FIG. 6. (Color online) Schematic diagram illustrating electron and ion paths in the 3-panel microion source. To the right of the grid, the ions travel to the collector. To the left of the grid, the ions travel back to the cathode.

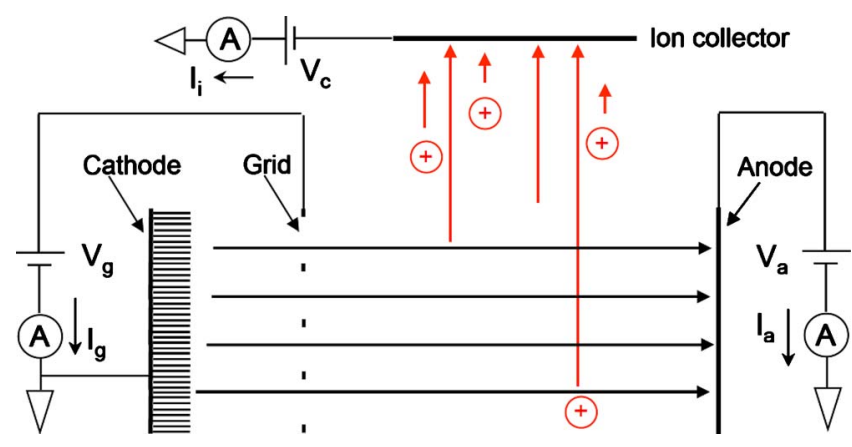

FIG. 7. (Color online) Schematic diagram illustrating predicted electron and ion paths in 4-panel micro ion source device.

the anode because of the positive potential applied to the grid and anode. Typically, the cathode was grounded, the grid was kept at $200 \mathrm{~V}$ and the anode was kept at $400 \mathrm{~V}$. The grid was biased at $200 \mathrm{~V}$ in order to obtain sufficient, stable electron current from the CNT cathode. It is then necessary to operate the anode in the 200-400 eV range in order to collect the electrons at the anode and operate the 4-panel device as a triode with an ion collector panel. Thus the energies of the electrons as they traveled from the grid to the anode were in the 200-400 eV range. Although this energy does not provide the highest possible ionization cross-section, these electrons nonetheless create ions via EI. These ions were then captured at the ion collector. A schematic illustrating this ionization setup is shown in Fig. 7.

\section{Test equipment}

The characterization of the microion source device was carried out at room temperature in a stainless steel vacuum chamber with a base pressure of $10^{-6}$ Torr. A calibrated capacitance manometer was used for pressure measurement to minimize gas-dependent effects. The manometer was designed for operation from $10^{-4}$ to 0.1 Torr. A roughing pump and turbo pump were used to achieve the required vacuum levels. A leak valve was used to controllably introduce the test gas into the characterization chamber. A Keithley 4200 Semiconductor Characterization System was used to characterize the performance of the microion source devices. The Keithley 4200 has six source-measure units (SMUs), each capable of sourcing voltages up to $\pm 210 \mathrm{~V}$ and measuring currents as low as femtoamperes. One of the six SMUs was specifically built for low-current applications and was equipped with a preamplifier. This SMU was used to measure the ion currents. For high-voltage measurements, a Keithley 2410 High-Voltage SourceMeter was used. It was capable of sourcing voltages up to $\pm 1100 \mathrm{~V}$ and measuring currents as low as $10 \mathrm{pA}$.

\section{B. Device characterization results}

\section{Results for the 3-panel microion source}

To achieve EI, the cathode was grounded while a positive voltage was applied to the grid and a negative bias applied to the ion collector (formerly anode). The cathode, grid and ion collector currents were recorded while the voltage applied to the grid was varied. Helium, argon, and xenon 


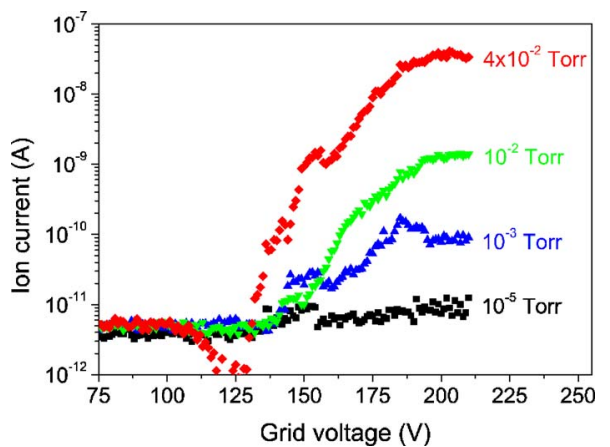

FIG. 8. (Color online) Ion current $\left(I_{i}\right)$ vs grid voltage. The legend indicates the helium chamber pressure for each data set. The ion collector voltage $\left(V_{i}\right)$ was held at $-210 \mathrm{~V}$ during these measurements.

were used to characterize the ion source. Figure 8 shows the measured ion current $\left(I_{i}\right)$, in this case helium ions, versus applied grid voltage. The ion current increases with pressure as the electron-neutral collision probability also increases. At the chamber base pressure of $10^{-3}$ mTorr, the measured ion collector current is $30 \mathrm{pA}$, while at a pressure of $50 \mathrm{mTorr}$ the ion current approaches $100 \mathrm{nA}$, representing a change in almost four orders of magnitude. Figure 9 shows the measured normalized ion current $\left(I_{i} / I_{g}\right)$, where $\left(I_{g}\right)$ is the measured grid current, versus pressure for helium, argon, and xenon obtained in the 3-panel microion source. The grid voltage $\left(V_{g}\right)$ was set to establish $1 \mu \mathrm{A}$ of measured grid current $\left(I_{g}\right)$, and the ion collector voltage $\left(V_{i}\right)$ was biased to $-210 \mathrm{~V}$. The grid and ion currents were recorded along with the pressure from the capacitance manometer while gas was slowly introduced into the chamber. The ion current recorded at the collector increased linearly with gas pressure, as expected from EI theory.

\section{Results for the 4-panel microion source}

A positive voltage was applied to the grid and anode electrodes to attract electrons to the anode. A small negative voltage was applied to the ion collector electrode to ensure collection of the (positively charged) ions. Figure 10 shows ion current for helium plotted as a function of grid voltage at different pressures. As the grid voltage increases, electron current increases, and hence, ion current increases. A rise in ion current with pressure was observed, as expected from the

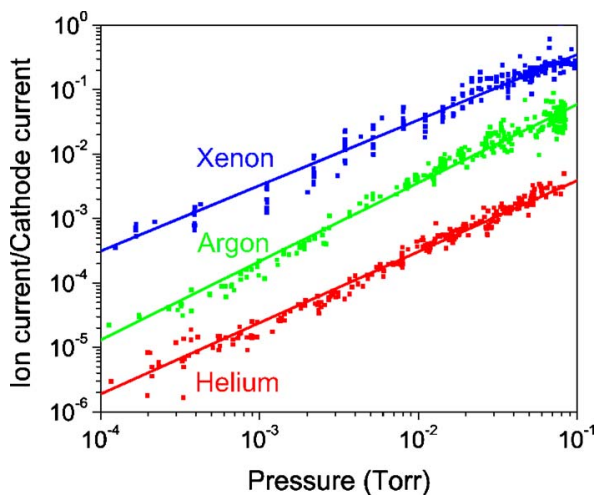

FIG. 9. (Color online) The measured ion collector current normalized by the measured electron grid current $I_{i} / I_{g}$ vs the measured chamber pressure.

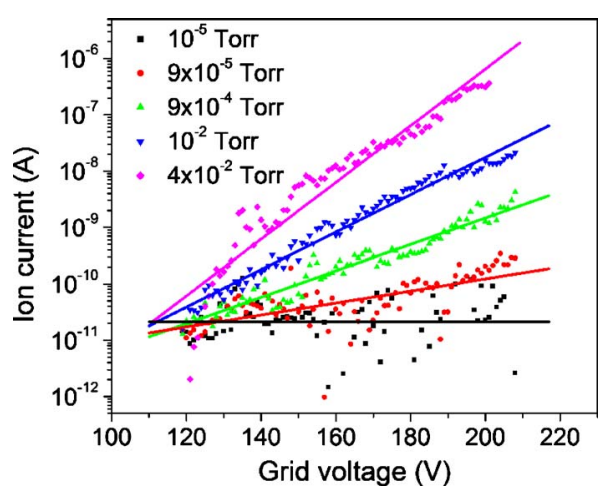

FIG. 10. (Color online) Ion current for helium vs grid voltage in the 4-panel microion source.

increasing number of species available for ionization with increasing pressure. Further increases in pressure ultimately create sufficient collisions and scattering events that the current begins to decrease. Figure 11 shows a plot of the ratio of ion current to cathode current, $I_{i} / I_{c}$, for helium, as a function of pressure for the 4-panel microion source (black squares) and the 3-panel microion source (red circles). The ion currents have been normalized by $I_{c}$ because $I_{i} \propto I_{c}$ in both cases, as is explained later. $I_{i} / I_{c}$ was observed to increase linearly with pressure. The 4-panel microion source shows (at least) a $10 \times$ improvement in $I_{i} / I_{c}$ compared to the 3-panel microion source at similar pressures.

\section{Comparison with commercial El ion sources}

The characteristics of a typical commercial EI source is given by Lambert. ${ }^{23}$ For a typical EI ion source, a current of $1 \times 10^{-10}$ A was considered typical. As seen in Fig. 8, for the three-panel ion source, and Fig. 10, for the four-panel ion source, the ion currents well exceed this level for pressures greater than $10^{-3}$ Torr and $10^{-4}$ Torr, respectively.

A more detailed discussion of the ion currents for both devices is included at the end of Sec. III D.

\section{Introduction to SIMION}

SIMION, an electron and ion optics software program, was used to simulate electron and ion paths in the microion sources. ${ }^{24,25}$ SIMION makes use of electric potential arrays that define the geometry and potentials of electrodes. The

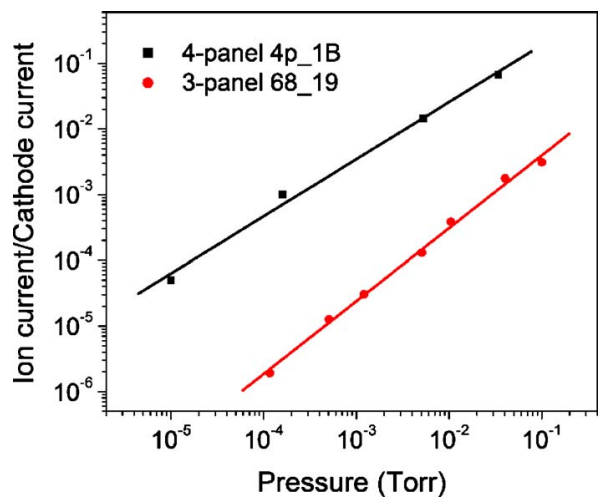

FIG. 11. (Color online) Ion current/cathode current for the 3-panel and 4-panel microion source devices as a function of pressure for helium. 
potentials at points in space between the electrodes are calculated by solving the Laplace equation by finite difference methods. ${ }^{24}$ The electrodes are used as boundary conditions to solve the Laplace equation. Once this is done, the potential arrays are projected as three-dimensional (3D) virtual images into an ion optics volume/workspace. Ions can be flown within the volume and their trajectories can be changed by changing the potentials applied to the electrodes. The Laplace equation assumes that there are no space charge effects. However, SIMION employs charge repulsion methods that can estimate certain types of space charge and particle repulsion effects. ${ }^{24}$ SIMION was used in this study to gain a better understanding of electron and ion paths and their dependence on device geometry and applied potentials of the device structures fabricated and characterized experimentally. As with any simulation program, the results obtained from SIMION may not represent the precise behavior of the miniature ion source devices. However, SIMION was a valuable aid in understanding the general behavior of the microion source devices, and optimizing the designs for improved performance.

A 3D drawing of the 3-panel and 4-panel microion source device to be simulated was made using SolidWorks, a $3 \mathrm{D}$ mechanical design and CAD software. ${ }^{26}$ The drawings were then imported into SIMION. The voltage on each electrode could be varied to simulate different operating conditions for the 3-panel and 4-panel microion source.

\section{Comparison of performance of the 4-panel microion source versus the 3-panel microion source}

This section provides a comparison of the various factors affecting the performance of the two microion sources. According to Smith and Tate, the ion current in an ion source is given by the following equation:

$$
\frac{I_{i}(E)}{I_{e}(E)}=\rho L \sigma(E),
$$

where $I_{i}(E)$ is the measured ion current, $I_{e}(E)$ is the measured electron current, $E$ is the kinetic energy of the electron beam, $\rho$ is the number density of the target gas, $L$ is the path length the electrons travel from which the ions are generated/ collected, and $\sigma(E)$ is the total EI cross-section of the gas. ${ }^{2}$ Thus, for a specific ion source, at a given pressure and test gas, the factors that affect ion current are the electron current contributing to ionization, electron energy of the colliding electron, electron path lengths and collector efficiency of the ion source. Representative 3-panel and 4-panel microion sources were chosen for the comparison that follows. (Sample designations 4-panel_1B and 3-panel 68_16 were chosen but they are simply referred to as the 3-panel and 4-panel microion sources, respectively, for the purposes of this paper). The voltages applied to various electrodes are listed in Table I.

\section{Electron current contributing to ionization}

In the 3-panel microion source, the grid is kept at a positive bias while the ion collector is kept at a negative bias with respect to the cathode. Some of the electron current
TABLE I. The voltages applied to different electrodes for the 3-panel and 4-panel microion source devices.

\begin{tabular}{lcccc}
\hline \hline Device & $\begin{array}{c}V_{\text {cathode }} \\
(\mathrm{V})\end{array}$ & $\begin{array}{c}V_{\text {grid }} \\
(\mathrm{V})\end{array}$ & $\begin{array}{c}V_{\text {anode }} \\
(\mathrm{V})\end{array}$ & $\begin{array}{c}V_{\text {ion collector }} \\
(\mathrm{V})\end{array}$ \\
\hline 3-panel & -100 & 210 & N.A. & -210 \\
4-panel & -200 & -3 & 210 & -210 \\
\hline \hline
\end{tabular}

emitted from the cathode is captured at the grid, while the remainder passes through the openings in the grid. These electrons travel a certain distance past the grid, are repelled by the negative bias applied to the ion collector, and turn back toward the grid where they are eventually captured (see Fig. 6). In the region between the grid and the ion collector, these electrons generate ions via EI. Thus, the electron current contributing to ionization, $I_{e}$, in the 3-panel microion source is the current in the region between the grid and the ion collector. However, the entire cathode current is eventually (either directly from the cathode or once the electrons pass through the grid and return) captured at the grid in this arrangement. The electrons that return either curve and strike the solid portion of the grid panel, or oscillate and strike the grid itself. The cathode current is identical to the grid current to three significant figures, as measured on separate ammeters (within the error limit of the ammeters). Thus, there was no way to experimentally measure $I_{e}$ in this configuration. Also, since there is no accurate way to simulate the field emission behavior in SIMION, it was difficult to extract a meaningful estimate for $I_{e}$ in the 3-panel and 4-panel microion sources from simulations.

In the 4-panel microion source, the triode portion of the device (i.e., cathode, grid and anode) is typically operated in the saturation region. Operating the device in the saturation region ensures that a known value of anode current is obtained for anode voltages beyond a certain threshold. A fraction of the electron current is captured by the grid and the remainder passes through the apertures in the grid and is captured at the anode (see Fig. 7).

The electrons contributing to ionization, $I_{e}$, are those traveling between the grid and the anode. Under vacuum and assuming ideal conditions, the electron current contributing to ionization, $I_{e}$, is given by $\left(I_{c}-I_{g}\right)=I_{a}$, which is the anode current. In practice, at higher pressures, $I_{a}<\left(I_{c}-I_{g}\right)$ because of imperfect collection of electrons at the anode, which may be the result of electron scattering. Secondary electrons generated during ionization can also artificially increase $I_{a}$. The value $\left(I_{c}-I_{g}\right)$ can be used as an approximation of $I_{e} . I_{c}$ and $I_{g}$ are also affected to some extent by secondary electrons generated during ionization. However, the effect of secondary electrons/collisions is most significant in the case of $I_{a}$ compared to $I_{c}$ and $I_{g}$ because of the greater distance traveled by electrons before reaching the anode. $I_{e}$ is typically between $50 \%$ and $90 \%$ of $I_{c}$, depending on device design. $I_{e}$ was approximately $0.6 I_{c}$ in the 4-panel microion source. In comparison, while an estimation of $I_{e}$ in the 3-panel microion source is difficult, work by Hyatt indicates that for charged particles, grids pass approximately the percentage of open space. ${ }^{27}$ Since the open space in the 3-panel and 4-panel 


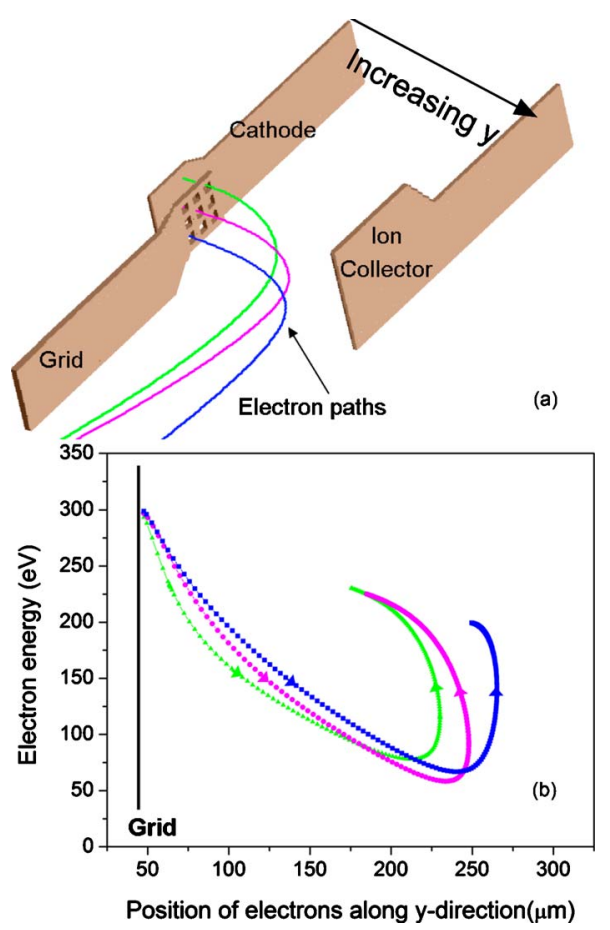

FIG. 12. (Color online) Electron paths and electron energies in the 3-panel microion source. (a) SIMION simulation showing electron paths of three electrons. The arrows denote direction of motion. (b) Electron energy as a function of position $(y)$ for the three electrons shown in (a).

grids is similar and the operating conditions are similar, it is reasonable to assume that the grids capture a similar amount of the electron current during the electrons initial approach.

\section{Electron energies and ionization cross- sections}

The electron energies in the microion source devices are critical in determining the measured ion currents because the ionization cross-section of each gas is a function of electron energy. Experimental measurement of electron energies was beyond the scope of this study, therefore, these energies were simulated using SIMION software. For purposes of this study, we believe this approach is valid because SIMION has been shown to provide a good approximation of electron energies measured under similar conditions. ${ }^{24,25}$ The electron energies at ten locations along the path of a typical electron originating from the field emission cathode were obtained via SIMION simulations. The ionization cross-sections for the gas of interest at those primary electron energies were then obtained from the literature. ${ }^{28}$ For the purposes of this analysis, helium was used as the test gas. The EI cross-sections $(\sigma)$ for helium at those ten electron energies were recorded. An average value for $\sigma_{\mathrm{He}}(E)$ in the 3-panel and 4-panel devices was then obtained by computing the average of the $\sigma(E)$ 's obtained at the ten locations. An average value for the electron energy was similarly obtained by computing the average of the electron energies at the ten locations.

Figure 12(a) shows a SIMION simulation illustrating the path of three electrons emitted from three different regions of the cathode in the 3-panel microion source. The voltages applied to the electrodes were the same values used in the experimental tests conducted on the standard microtriode,
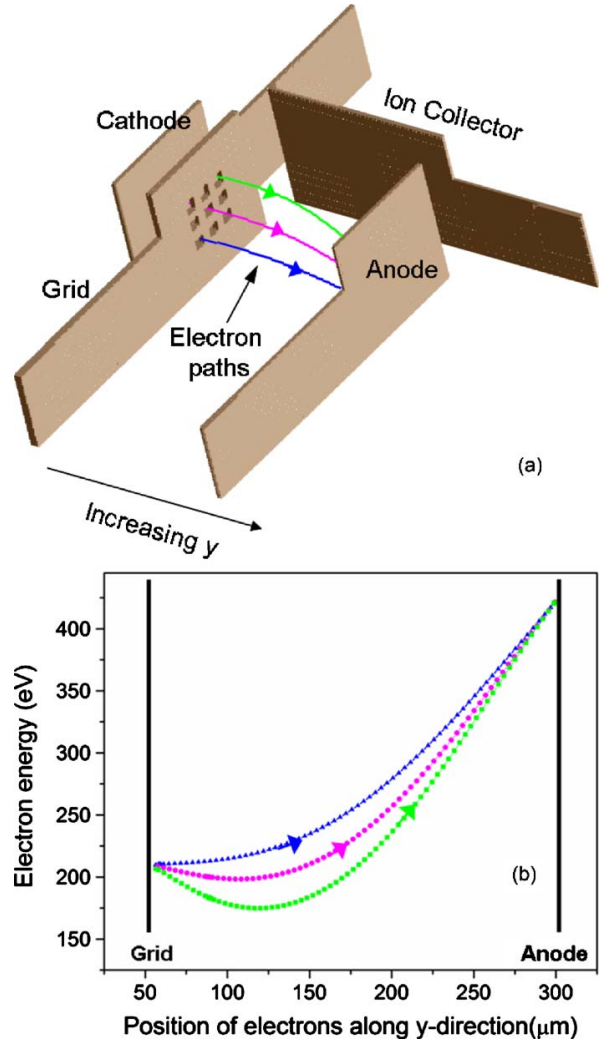

FIG. 13. (Color online) Electron paths and electron energies in the 4-panel microion source. (a) SIMION simulation showing electron paths of three electrons. The arrows denote direction of motion. (b) Electron energy as a function of position $(y)$ for the three electrons shown in (a). The dip in electron energy for all three trajectories is due to the negative bias voltage of the ion collector panel. Closer electrons show a larger dip in energy.

3-panel microion source. As expected, a fraction of the electrons pass through the grid, travel toward the ion collector and after a certain distance, turn back toward the grid. Figure 12(b) plots the electron energies in the 3-panel microion source as a function of their position along the $y$-axis. The electron energies in the 3-panel device ranged from 50 to 300 $\mathrm{eV}$. (The highest value of $\sigma_{\mathrm{He}}(E)$ is $0.37 \times 10^{-16} \mathrm{~cm}^{2}$ and occurs in the $125-130 \mathrm{eV}$ range.) The average electron energy within the device area was calculated to be approximately $159 \mathrm{eV}$. Thus, the average value of $\sigma_{\mathrm{He}}(E)$ in the 3-panel microion source was $0.35 \times 10^{-16} \mathrm{~cm}^{2}$, which is $92 \%$ of the peak value.

Figure 13(a) shows a SIMION simulation illustrating the path of three electrons in the 4-panel microion source. The electrons follow almost a straight path from the grid to the anode. Figure 13(b) plots the electron energies in the 4-panel microion source as a function of their position along the $y$-axis. The electron energies in the device ranged from 175 to $300 \mathrm{eV}$. Note that the dip in electron energy in the range of $75-150 \mu \mathrm{m}$ past the grid is caused by the negative field from the ion collector panel. The average energy of electrons in the device was calculated to be approximately $260 \mathrm{eV}$. The average value of $\sigma_{\mathrm{He}}(E)$ in the 4-panel device was calculated to be $0.31 \times 10^{-16} \mathrm{~cm}^{2}$, which is $83 \%$ of the peak value. Thus, the ionization average cross-section for helium was approximately $12 \%$ higher in the 3-panel microion source, compared to the 4-panel microion source. This is because the 
range of electron energies in the 3-panel microion source includes 125 to $130 \mathrm{eV}$ (the energy range at which ionization cross-section peaks) in two sections along the path of each electron.

\section{Electron path length}

The electron path length is defined to be the length of the electron path along which ions are collected. ${ }^{2}$ The electron path length for the 3-panel microion source is defined to be the distance traveled by the electron beam after passing through the openings in the grid and before leaving the device area and was calculated to be approximately $500 \mu \mathrm{m}$ from SIMION simulations. Such a long electron path was obtained because the electrons initially travel toward the ion collector and the electron beam is then bent by the retarding potential applied to the ion collector, as shown in Fig. 12. The electron path length in the 4-panel device was calculated to be $260 \mu \mathrm{m}$ from SIMION simulations. This was approximately equal to the distance between the grid and anode electrodes, because the electron beam follows essentially a straight path from the grid to the anode, as seen in Fig. 13. Thus, the electron path length in the 3-panel microion source was almost twice that in the 4-panel device.

\section{Ion collection efficiency}

All the factors discussed previously affect the number of ions generated in the microion source devices. However, the collection of the ions created in the device is also a significant factor in determining the ion current that is measured in the device. The ion collection efficiency can be defined as the ratio of the number of ions collected to the number of ions created. The placement of the ion collector electrode relative to the other electrodes in the device and the voltages applied to the different electrodes play a major role in determining the collection efficiency. With regards to the collection efficiency of the 3-panel device, as can be seen in Fig. 14(a), the ion paths in the three-panel device are not confined to the grid to collector region, leading to a possible overestimation of the ion collection efficiency. However, this does not change the conclusion of the relative efficiencies of the two devices. If the ion collector on the 3-panel device extended to the end of the grid panel, the electrons should turn around as shown in Fig. 6. Although complications caused by panel support structures will need to be considered, this might result in a more efficient ionization source for the 3-panel configuration and will be considered for future designs. For this calculation, 100 hydrogen ions (mass $=1 \mathrm{amu}$, charge $=+1$ ) were used. Their initial energy was assumed to be $0 \mathrm{eV}$. The ions were all created at various points along the different electron paths predicted by SIMION.

Figure 14(a) shows a SIMION simulation illustrating electron and ion paths in the 3-panel microion source. The ion collection efficiency was calculated to be approximately $45 \%$ from SIMION. The main reason for the low ion collection efficiency is the poor alignment of the grid and ion collector electrode panels. Due to this arrangement of electrodes and the voltages applied to the electrodes, the electron beam was bent in such a way that it traveled away from the ion collec-

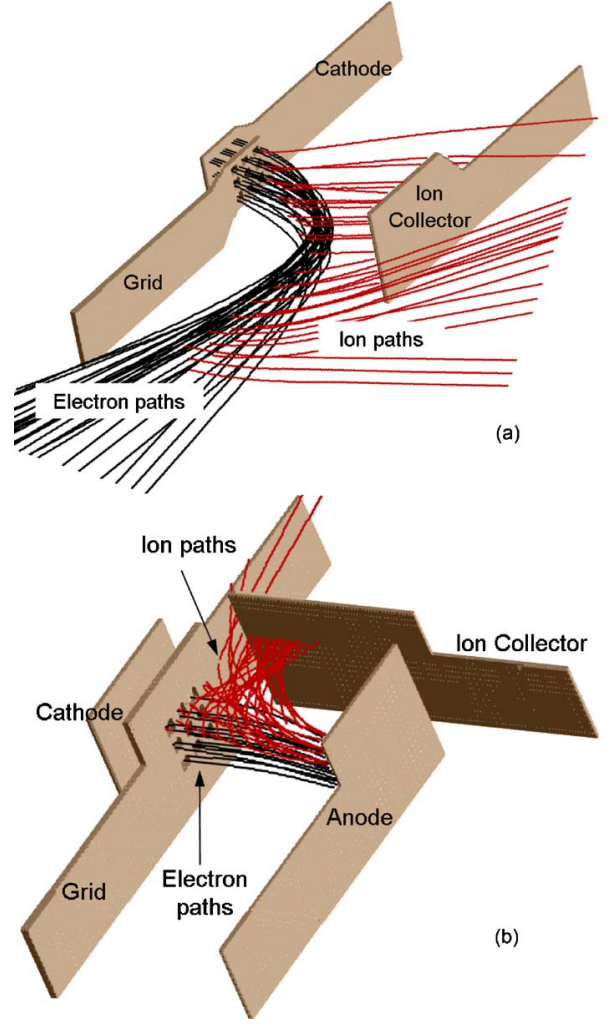

FIG. 14. (Color online) SIMION simulations illustrating ion collector efficiencies. (a) SIMION simulation illustrating ion collector efficiency in the 3-panel microion source. (b) SIMION simulation illustrating ion collector efficiency in the 4-panel microion source device.

tor panel. Most of the ions created by the electrons during their flight from the grid to the ion collector were captured at the ion collector. But the ions created by the electrons after their paths were bent could not be captured at the ion collector, as shown in Fig. 14(a). The electric field lines in this region were such that those ions traveled past the ion collector panel and away from the device.

Figure 14(b) shows a SIMION simulation illustrating electron and ion paths in the 4-panel microion source. The ion collection efficiency was calculated to be approximately $90 \%$ from SIMION. The main reason for the high ion collection efficiency is the shape of the electron beam and the placement of the ion collector almost parallel to the electron beam. This ensures that ions created almost everywhere along the electron path are collected at the ion collector electrode. Also, the ion collector electrode was situated only $50 \mu \mathrm{m}$ away from the edges of the grid and anode electrodes, which reduced the distance the ions traveled before being captured at the collector. This ensured that only a small percentage of ions escaped through the space between the ion collector and cathode electrodes, as shown in Fig. 14(b). The ions that escaped were mostly created in that part of the ionization volume that was closest to the anode, where the electrons typically had high energies of 350 to $400 \mathrm{eV}$. It should be noted that ionization cross-section decreases with electron energy for high electron energies (i.e., greater than $150 \mathrm{eV}$ ) for most gases. Thus, it is believed that the actual ion collection efficiency is even greater than the $90 \%$ value calculated herein for the 4-panel microion sources. Future 
TABLE II. Comparison of important factors that affect ion current in the 3-panel and 4-panel microion sources. As can be seen in Fig. 14(a), the ion paths in the three-panel device are not confined to the grid-to-collector region, leading to a possible overestimation of the ion collection efficiency.

\begin{tabular}{lccccc}
\hline \hline Device & $\begin{array}{c}\text { Electron } \\
\text { energies } \\
(\mathrm{eV})\end{array}$ & $\begin{array}{c}\text { Average ionization } \\
\text { cross-section } \\
\left(10^{-16} \mathrm{~cm}^{2}\right) \\
\text { for helium }\end{array}$ & $\begin{array}{c}\text { Ion collection } \\
\text { efficiency } \\
(\%)\end{array}$ & $\begin{array}{c}\text { Electron path } \\
\text { length } \\
(\mu \mathrm{m})\end{array}$ & $\begin{array}{c}\text { Ratio of ion current to } \\
\text { electron current at 50 mTorr }\end{array}$ \\
\hline 3-panel & 50 to 300 & 0.35 & 45 & 500 & $2.1 \times 10^{-3}$ \\
4-panel & 175 to 300 & 0.31 & 90 & 250 & $2.3 \times 10^{-2}$ \\
\hline \hline
\end{tabular}

research will include the modeling of these and other ion source configurations, taking into account integrated ion cross sections as a function of electron energies, giving a more accurate representation of ionization probability throughout the device as well as collection efficiency and thereby enable further improvements in source design.

Thus far, the different factors that affect the measured ion current in the 3-panel and 4-panel microion source devices have been compared and analyzed. Comparison of the measured ion currents for these two ion source geometries is discussed next. The ratio of ion current to cathode current, $\left(I_{i} / I_{c}\right)$, was chosen as a measure of performance in order to minimize the impact of intrinsic differences in field emission properties of the cathode from one device to the next and enable the analysis to focus on the effects of device geometry. $I_{c}$ was chosen for this normalization because the actual values of electron current contributing to ionization, $I_{e}$, could not be measured for both devices. In both ion sources, $I_{e}$ is proportional to $I_{c}$ as was discussed earlier. Thus, the cathode current was used to normalize the measured ion current and enable comparisons of ion source performance for the different microion source geometries.

Figure 11 shows the normalized ion current, $I_{i} / I_{c}$, as a function of pressure for the 4-panel and 3-panel microion source devices. A rise in $I_{i} / I_{c}$ with pressure was seen for both devices. $I_{i} / I_{c}$ for the 4-panel device was higher than $I_{i} / I_{c}$ for the 3-panel device at similar pressures. The main factors believed to be responsible for this are the increased electron current contributing to ionization and the higher ion collection efficiency in the 4-panel device as discussed previously. The main factors affecting ion currents in both devices are listed in Table II.

The electron path length and average ionization crosssection are higher in the 3-panel device but their combined effect on $\left(I_{i} / I_{c}\right)$ still falls short of the combined effect of the larger $I_{e}$ and higher collection efficiency of the 4-panel device.

\section{E. Increasing the efficiency of the 4-panel microion source}

The efficiency of the 4-panel device can be increased further by changing device design and geometry of the MWNTs used as the field emission source. To maximize the number of ions generated per primary electron, the electron energy should be in the 70 to $150 \mathrm{eV}$ range. This can be achieved by operating the grid electrode at $70 \mathrm{~V}$ and the anode at $150 \mathrm{~V}$. At least $1 \mu \mathrm{A}$ of primary electron current is necessary to operate the ion source, and this was not achieved at a grid bias of $70 \mathrm{~V}$ (with respect to the cathode) with the existing set of devices. Further, the device design should be such that most of the electron current emitted by the cathode travels from the cathode (through the grid) to the anode, when the grid is biased at $70 \mathrm{~V}$ and the anode is biased at $150 \mathrm{~V}$. To achieve this, certain changes in device design are necessary. First, the turn-on field for field emission from the MWNTs should be lowered so that at least $1 \mu \mathrm{A}$ of electron current is achieved at a grid bias of $70 \mathrm{~V}$. This can be done by reducing the cathode-to-grid spacing in combination with optimizing the MWNT pattern on the cathode. Reducing the cathode-to-grid spacing will allow higher fields to be achieved at lower voltages, since the electric field is proportional to (1/distance). It has been shown that reducing the density of MWNTs in the bundles is one way to decrease the turn-on field of the MWNT film. ${ }^{29}$ In addition, Fujii et $a .^{30}$ reported that optimizing the length of MWNT bundles with respect to the distance between bundles reduced the turn-on field significantly. This finding can be used to modify the MWNT pattern (and length) on the cathode of the microion source devices. It is also believed that most of the field emission from a MWNT bundle occurs from the edges of the MWNT bundle rather than the entire bundle. ${ }^{31}$ The MWNT pattern of the microion source devices can be modified to maximize the perimeter (of the pattern) for the same area, as this is expected to decrease the field emission turn-on voltage. Further, the grid-to-anode spacing can be increased since this will increase the electron path length and hence, the number of ions created.

\section{SUMMARY}

In summary, the authors present a detailed comparison of two designs of microscale EI sources. Both ion sources utilized the field emission properties of CNTs and were fabricated using MEMS fabrication techniques. The 4-panel design was found to perform $10 \times$ better than the 3-panel design. Experimental results along with SIMION simulations were used to evaluate the improved performance of the 4-panel device. The electron current contributing to ionization and the ion collection efficiency are thought to be the main factors that contribute to this performance enhancement. Suggestions for further improvements in microscale ionization source design are provided based on the understanding gained by this integrated evaluation of experimental results and simulations. 


\section{ACKNOWLEDGMENTS}

The authors acknowledge Dr. Chris Bower and Dr. Scott Wolter for their technical contributions at various stages of this study. This work was supported by Grants ECCS0428540 and ECCS-0801942 from the National Science Foundation and Grant No. NNL05AA14A from NASA.

${ }^{1}$ Physics and Technology of Ion Sources, 2nd ed., edited by I. G. Brown (Wiley-VCH, Weinheim, Germany, 2004).

${ }^{2}$ L. J. Kieffer and G. H. Dunn, Rev. Mod. Phys. 38, 1 (1966).

${ }^{3}$ C. A. Bower, K. H. Gilchrist, J. R. Piascik, B. R. Stoner, S. Natarajan, C. B. Parker, S. D. Wolter, and J. T. Glass, Appl. Phys. Lett. 90, 124102 (2007).

${ }^{4}$ S. Natarajan, C. B. Parker, J. T. Glass, J. R. Piascik, K. H. Gilchrist, C. A. Bower, and B. R. Stoner, Appl. Phys. Lett. 92, 224101 (2008).

${ }^{5}$ S. Natarajan, K. H. Gilchrist, J. R. Piascik, C. B. Parker, J. T. Glass, and B. R. Stoner, Appl. Phys. Lett. 94, 044109 (2009).

${ }^{6}$ O. Kornienko, P. T. A. Reilly, W. B. Whitten, and J. M. Ramsey, Anal. Chem. 72, 559 (2000).

${ }^{7}$ C.-W. Baik, Y.-M. Son, S. Kim II, S. C. Jun, J.-S. Kim, J. Hwang, J.-M. Kim, S.-W. Moon, H. J. Kim, J.-K. So, and G.-S. Park, Microfabricated Coupled-Cavity Backward-Wave Oscillator for Terahertz Imaging (Inst. of Elec. and Elec. Eng. Computer Society, Monterey, California, 2008).

${ }^{8}$ J. B. Cho, H. J. Yoon, K. W. Jung, S. S. Yang, K. H. Koh, and S. Lee, Micro Mass Spectrometer Using Triode Electron Emitters with a Planar Carbon-Nanoparticle Cathode as Ion Source (Inst. of Elec. and Elec. Eng. Computer Society, Bangkok, Thailand, 2007).

${ }^{9}$ L. Y. Chen, L. F. Velásquez-García, X. Wang, K. Cheung, K. Teo, and A. I. Akinwande, "Design, fabrication and characterization of double-gated vertically aligned carbon nanofiber field emitter arrays," Technical Digest of the 20th International Vacuum Nanoelectronics Conference, Chicago, IL, 2007, pp. 82-83.

${ }^{10}$ W. Zhu, C. Bower, O. Zhou, G. Kochanski, and S. Jin, Appl. Phys. Lett. 75, 873 (1999).
${ }^{11}$ K. A. Dean and B. R. Chalamala, Appl. Phys. Lett. 76, 375 (2000).

${ }^{12}$ C. Dong and G. R. Myneni, Appl. Phys. Lett. 84, 5443 (2004).

${ }^{13}$ I. M. Choi and S. W. Woo, Appl. Phys. Lett. 87, 173104 (2005).

${ }^{14}$ T. T. King, S. A. Getty, P. A. Roman, F. A. Herrero, H. H. Jones, D. M. Kahle, B. Lynch, G. Suarez, W. B. Brinckerhoff, and P. R. Mahaffy, Simulation of a Miniature, Low-Power Time-of-Flight Mass Spectrometer for In Situ Analysis of Planetary Atmospheres (SPIE, Orlando, Florida, 2008).

${ }^{15}$ L. F. Velasquez-Garcia and A. I. Akinwande, A PECVD CNT-based open architecture field for portable mass spectrometry, in 21 IEEE International Conference on Micro Electro Mechanical Systems (MEMS 2008), 2008, Tucson, Arizona.

${ }^{16}$ J.-P. Hauschild, E. Wapelhorst, and J. Müller, Int. J. Mass. Spectrom. 264 53 (2007).

${ }^{17}$ E. Wapelhorst, J.-P. Hauschild, and J. Müller, Sens. Actuators, A 138, 22 (2007).

${ }^{18}$ J. Carter, A. Cowen, B. Hardy, R. Mahadevan, M. Stonefield, and S. Wilcenski, The PolyMUMPS Design Handbook, (Rev. 11.0). 2005.

${ }^{19}$ H. Cui, O. Zhou, and B. R. Stoner, J. Appl. Phys. 88, 6072 (2000).

${ }^{20}$ C. A. Bower, O. Zhou, W. Zhu, D. J. Werder, and S. Jin, Appl. Phys. Lett. 77, 2767 (2000).

${ }^{21}$ S. Natarajan, Electrical and Computer Engineering (Duke University, Durham, North Carolina, 2008), p. 199.

${ }^{22}$ J. T. Tate and P. T. Smith, Phys. Rev. 39, 270 (1932).

${ }^{23}$ J. B. Lambert, Organic Structural Spectroscopy, 6th ed. (Prentice Hall, Upper Saddle River, New Jersey, 1998).

${ }^{24}$ Scientific Instrument Services Inc., SIMION v8, 2007.

${ }^{25}$ J. Kipritidis, M. Fitzgerald, and J. Khachan, J. Phys. D 40, 5170 (2007).

${ }^{26}$ M. Majumder, N. Chopra, and B. J. Hinds, J. Am. Chem. Soc. 127, 9062 (2005).

${ }^{27}$ J. M. Hyatt, Phys. Rev. 32, 922 (1928).

${ }^{28}$ D. Rapp and P. Englander-Golden, J. Chem. Phys. 43, 1464 (1965).

${ }^{29}$ S. H. Jo, Y. Tu, Z. P. Huang, D. L. Carnahan, D. Z. Wang, and Z. F. Ren, Appl. Phys. Lett. 82, 3520 (2003).

${ }^{30}$ S. Fujii, S.-I. Honda, H. Kawai, K. Ishida, K. Oura, and M. Katayama, Diamond Relat. Mater. 17, 556 (2008).

${ }^{31}$ T.-W. Weng, Y.-H. Lai, and K.-Y. Lee, Appl. Surf. Sci. 254, 7755 (2008). 